OPEN ACCESS

Edited by:

Andrea Sanson,

Dipartimento di Fisica e Astronomia,

Università degli Studi di Padova, Italy

Reviewed by:

Lars Gundlach,

University of Delaware, United States

Titus Sebastiaan Van Erp,

Norwegian University of Science and

Technology, Norway

*Correspondence:

Peng Tong

tongpeng@issp.ac.cn

Specialty section:

This article was submitted to

Physical Chemistry and Chemical

Physics,

a section of the journa

Frontiers in Chemistry

Received: 11 January 2018 Accepted: 06 March 2018

Published: 21 March 2018

Citation:

Guo $X$, Tong $P$, Lin J, Yang C, Zhang $K$, Lin S, Song $W$ and Sun $Y$ (2018) Effects of Cr Substitution on

Negative Thermal Expansion and Magnetic Properties of Antiperovskite

$\mathrm{Ga}_{1-x} \mathrm{Cr}_{x} \mathrm{~N}_{0.83} \mathrm{Mn}_{3}$ Compounds.

Front. Chem. 6:75.

doi: 10.3389/fchem.2018.00075

\section{Effects of Cr Substitution on Negative Thermal Expansion and Magnetic Properties of Antiperovskite $\mathrm{Ga}_{1-x} \mathrm{Cr}_{\mathbf{x}} \mathrm{N}_{0.83} \mathrm{Mn}_{3}$ Compounds}

\author{
Xinge Guo ${ }^{1,2}$, Peng Tong ${ }^{2 *}$, Jianchao Lin $^{2}$, Cheng Yang ${ }^{2}$, Kui Zhang ${ }^{2}$, Shuai Lin ${ }^{2}$, \\ Wenhai Song ${ }^{2}$ and Yuping Sun ${ }^{2,3,4}$ \\ ${ }^{1}$ School of Science, Hebei University of Science and Technology, Shijiazhuang, China, ${ }^{2}$ Key Laboratory of Materials Physics, \\ Institute of Solid State Physics, Chinese Academy of Sciences, Hefei, China, ${ }^{3}$ High Magnetic Field Laboratory, Chinese \\ Academy of Sciences, Hefei, China, ${ }^{4}$ Collaborative Innovation Center of Advanced Microstructures, Nanjing University, \\ Nanjing, China
}

Negative thermal expansion (NTE) and magnetic properties were investigated for antiperovskite $\mathrm{Ga}_{1-x} \mathrm{Cr}_{x} \mathrm{~N}_{0.83} \mathrm{Mn}_{3}$ compounds. As $x$ increases, the temperature span $(\Delta T)$ of NTE related with $\Gamma^{5 \mathrm{~g}}$ antiferromagnetic (AFM) order is expanded and shifted to lower temperatures. At $x=0.1$, NTE happens between 256 and $318 \mathrm{~K}(\Delta T=62 \mathrm{~K})$ with an average linear coefficient of thermal expansion, $\alpha_{L}=-46 \mathrm{ppm} / \mathrm{K}$. The $\Delta T$ is expanded to $81 \mathrm{~K}(151-232 \mathrm{~K})$ in $x=0.2$ with $\alpha_{L}=-22.6 \mathrm{ppm} / \mathrm{K}$. Finally, NTE is no longer visible for $x \geq 0.3$. Ferromagnetic order is introduced by $\mathrm{Cr}$ doping and continuously strengthened with increasing $x$, which may impede the AFM ordering and thus account for the broadening of NTE temperature window. Moreover, our specific heat measurement suggests the electronic density of states at the Fermi level is enhanced upon Cr doping, which favors the FM order rather than the AFM one.

Keywords: negative thermal expansion, antiferromagnetic order, specific heat, antiperovskite compounds, Cr substitution

\section{INTRODUCTION}

Negative thermal expansion (NTE) materials, which contract upon heating, have received great attentions recently (Mary et al., 1996; Takenaka and Takagi, 2005; Goodwin et al., 2008; Long et al., 2009; Greve et al., 2010; Azuma et al., 2011; Yamada et al., 2011; Huang et al., 2013; Panda et al., 2014; Zhao et al., 2015). From the view point of applications, NTE materials can be used as fillers for compensating and controlling the positive thermal expansion (PTE) of normal materials by forming composites (Romao et al., 2003; Chen et al., 2015). NTE has been observed in many materials due to different mechanisms, including flexible framework in crystal structure (Mary et al., 1996; Goodwin et al., 2008; Greve et al., 2010; Ge et al., 2016; Hu et al., 2016; Jiang et al., 2016), ferroelectricity (Xing et al., 2003; Chen et al., 2013), charge transformation (Long et al., 2009; Azuma et al., 2011; Yamada et al., 2011), magnetovolume effect (MVE) (Takenaka and Takagi, 2005; Huang et al., 2013; Li et al., 2015, 2016), and martensitic transformation (Zhao et al., 2015; Lin et al., 2016). Among them, the NTE related with MVE in antiperovskite manganese nitrides $\mathrm{ANMn}_{3}$ (A: transition metal or semiconducting elements) has been extensively studied because of the large and isotropic 
NTE with tunable linear coefficient of thermal expansion $\left(\alpha_{L}\right)$, good mechanical properties (large Young's modulus and hardness) and thermal/electrical conductivities (Takenaka and Takagi, 2005; Sun et al., 2007; Huang et al., 2008; Song et al., 2011; Tong et al., 2013a,b; Tan et al., 2014).

Large lattice volume contraction of a few percent at the antiferromagnetic (AFM) to paramagnetic (PM) phase transition due to MVE has been reported in antiperovskite manganese nitrides decades ago (Fruchart and Bertaut, 1978). However, due to the limited temperature window (a few K) of MVE, these materials cannot be practically used as PTE compensators. In 2005, Takenaka firstly reported the broadening of MVE window in $\mathrm{Cu}_{1-x} \mathrm{Ge}_{x} \mathrm{NMn}_{3}$ (Takenaka and Takagi, 2005). From then on, many studies reported the NTE properties in $\mathrm{ANMn}_{3}(\mathrm{~A}=\mathrm{Zn}$, $\mathrm{Ga}, \mathrm{Ag}$, and $\mathrm{Cu}$ ) by substituting $\mathrm{A}$ with non-magnetic elements, such as Ge, Sn, Si (Sun et al., 2007, 2010a,b; Huang et al., 2008; Takenaka et al., 2008; Dai et al., 2014). Neutron diffraction studies indicated that the pronounced MVE occurs due to the ordering of the non-collinear triangular $\Gamma^{5 \mathrm{~g}}$ AFM spin configuration, and the non-magnetic element doping slows down the ordering of $\Gamma^{5 \mathrm{~g}}$ AFM phase (Iikubo et al., 2008a; Song et al., 2011; Deng et al., 2015a,b). Local structure measured via the neutron pair distribution function (PDF) (Iikubo et al., 2008b; Tong et al., 2013a) and x-ray absorption fine structure measurements (Matsuno et al., 2009) suggested a strong relation between the broadening of AFM transition and the local lattice distortions, though a detailed mechanism is still under debate (Tong et al., 2013a). Very recently, we found that by partially replacing A in $\mathrm{ANMn}_{3}$ (i.e., $\mathrm{GaN}_{0.8} \mathrm{Mn}_{3}, \mathrm{AgNMn}_{3}$ ) with $\mathrm{Mn}$, the $\mathrm{MVE}$ window was expanded as well (Guo et al., 2015; Lin et al., 2015), while local structural distortion was not observed (Guo et al., 2015). For example, in $\mathrm{Ga}_{1-x} \mathrm{Mn}_{x} \mathrm{~N}_{0.8} \mathrm{Mn}_{3}$ the $\Delta T$ of NTE reaches $54 \mathrm{~K}$ (between 255 and $309 \mathrm{~K}, \alpha_{L}=-42 \mathrm{ppm} / \mathrm{K}$ ) and $73 \mathrm{~K}$ (between 206 and $279 \mathrm{~K}, \alpha_{L}=-25 \mathrm{ppm} / \mathrm{K}$ ) for $x=0.25$ and 0.3 , respectively (Guo et al., 2015). Large NTE with $\alpha_{L} \sim-20$ $\mathrm{ppm} / \mathrm{K}$ at cryogenic temperatures (below $120 \mathrm{~K}$ ) was achieved in $\left(\mathrm{Ga}_{0.7} \mathrm{Cu}_{0.3}\right)_{1-x} \mathrm{Mn}_{x} \mathrm{NMn}_{3}$ with $x=0.25$ and 0.3 (Guo et al., 2017). In those Mn-doped compounds, in addition to the AFM order that gives rise to the large volume change, the coexisting FM order was demonstrated to impede the growth of the AFM order and thus cause the broadened $\Delta T$ of lattice contraction (Guo et al., 2015, 2017; Lin et al., 2015). It is interesting to check whether other $3 \mathrm{~d}$ elements can tune the NTE of $\mathrm{ANMn}_{3}$ as the Mn does.

Here, we report influences of $\mathrm{Cr}$ substitution for $\mathrm{Ga}$ on thermal expansion and magnetic properties of MVE-compound $\mathrm{GaN}_{0.83} \mathrm{Mn}_{3} . \mathrm{GaN}_{0.83} \mathrm{Mn}_{3}$ is AFM below $T_{\mathrm{N}} \sim 360 \mathrm{~K}$ (Kasugai et al., 2012). Upon substituting $\mathrm{Cr}$ for Ga, the AFM ground is quickly suppressed. Meanwhile, FM order is introduced and increasingly enhanced with increasing $\mathrm{Cr}$ doping level. Accompanying with the suppression of AFM state, the sharp MVE of the parent compound is quickly moved to lower temperatures and the related temperature range is widened. A quite large NTE temperature window of $81 \mathrm{~K}(151-232 \mathrm{~K})$ with a considerably large average $\alpha_{L} \sim-22.6 \mathrm{ppm} / \mathrm{K}$ was observed in $x=0.2$. The emergence of FM order can be attributed to the increasing electronic density of states (DOS) at the Fermi energy
$\left(\mathrm{E}_{\mathrm{F}}\right)$ as indicated by the increased electronic contribution to the specific heat at low temperatures.

\section{EXPERIMENTAL}

Polycrystalline samples $\mathrm{Ga}_{1-x} \mathrm{Cr}_{x} \mathrm{~N}_{0.83} \mathrm{Mn}_{3}(x=0,0.1,0.2$, $0.3,0.4)$ were prepared by direct solid state reaction with $\mathrm{Ga}$ ingot $(4 \mathrm{~N}), \mathrm{Cr}(3 \mathrm{~N}), \mathrm{Mn}(4 \mathrm{~N})$, and self-made $\mathrm{Mn}_{2} \mathrm{~N}$ powders. The starting materials were mixed in the desired proportions, sealed in evacuated quartz tubes $\left(10^{-3} \mathrm{~Pa}\right)$ and then annealed at $873-973 \mathrm{~K}$ for 5 days. After quenching the tubes to room temperature, the products were pulverized, mixed, pressed into pellets, and annealed again at $1,073-1,173 \mathrm{~K}$ for extra 8 days. The final samples were checked by X-ray diffraction (XRD) on a Bruker X-ray diffractometer (D8 Advance) with $\mathrm{Cu} \mathrm{K} \alpha$ radiations at room temperature. The magnetization measurements were performed on a Superconducting Quantum Interference Device Magnetometer (SQUID, Quantum Design). By using a strain gauge, linear thermal expansion $\Delta L / L$ was measured on a Physical Property Measurement System (PPMS, Quantum Design; Lin et al., 2015). On the same PPMS system, specific heat was measured for $x=0$ and 0.2 compounds.

\section{RESULTS AND DISCUSSION}

Figure 1 shows the room-temperature XRD patterns for $\mathrm{Ga}_{1-x} \mathrm{Cr}_{x} \mathrm{~N}_{0.83} \mathrm{Mn}_{3}(x=0,0.1,0.2,0.3,0.4)$ samples. All the samples are single-phase with a typical cubic antiperovskite structure (space group: Pm-3m), except for a very small amount of $\mathrm{CrN}$ detected in $x=0.4$. The (111) peak shifts toward higher angles as $x$ increases, which indicates the decrease of lattice constant with the increase of $\mathrm{Cr}$ content. Figure 2A presents the temperature dependent magnetization $M(T)$ of $\mathrm{Ga}_{1-x} \mathrm{Cr}_{x} \mathrm{~N}_{0.83} \mathrm{Mn}_{3}(0 \leq x \leq 0.3)$ measured at $H=100$ Oe under both zero-field-cooling (ZFC) and field-cooling (FC) modes. As shown in Figure 2B, there is a kink at $358 \mathrm{~K}$ for $x=0$, indicating an AFM to PM transition as often observed in antiperovskite manganese nitrides. This value agrees well with the Neel temperature $\left(T_{\mathrm{N}}\right)$ of $\mathrm{GaN}_{0.83} \mathrm{Mn}_{3}$ reported previously (Kasugai et al., 2012). When $x=0.1, T_{\mathrm{N}}$ is decreased to $318 \mathrm{~K}$. In slightly $\mathrm{Mn}$-doped $\mathrm{Ga}_{1-x} \mathrm{Mn}_{x} \mathrm{~N}_{0.8} \mathrm{Mn}_{3}$ (Guo et al., 2015), $\left(\mathrm{Ga}_{0.7} \mathrm{Cu}_{0.3}\right)_{1-x} \mathrm{Mn}_{x} \mathrm{~N}_{0.8} \mathrm{Mn}_{3}$ (Guo et al., 2017), and $\mathrm{Ag}_{1-x} \mathrm{Mn}_{x} \mathrm{NMn}_{3}$ (Lin et al., 2015), the $M(T)$ s are featured by a clear peak in the ZFC curves, while the related FC $M(T)$ s show a FM-like transition. This behavior was verified as a glassy transition (Guo et al., 2015, 2017; Lin et al., 2015). In contrast, for $x=0.1 \mathrm{Cr}$ doped sample, FM-like transition was observed at around $120 \mathrm{~K}$ in both ZFC and FC $M(T)$ curves with an obvious divergence between them at lower temperatures. The absence of peak in ZFC $M(T)$ curve is indicative of the emergence of long range FM order below $120 \mathrm{~K}$. For $x=0.2$, the FM-like transition is increased to $230 \mathrm{~K}$. The kink on ZFC $M(T)$ referring to $T_{\mathrm{N}}$ is no longer visible. Instead, a drop of magnetization happens at $210 \mathrm{~K}$ in both ZFC and FC $M(T)$ curves, similar to that observed in $\mathrm{Ga}_{1-x} \mathrm{Mn}_{x} \mathrm{~N}_{0.8} \mathrm{Mn}_{3}$ with $x=0.3$ (Guo et al., 2015). The $M(T)$ curves for $x=0.3$ display a FM transition at $322 \mathrm{~K}$, though the FC 


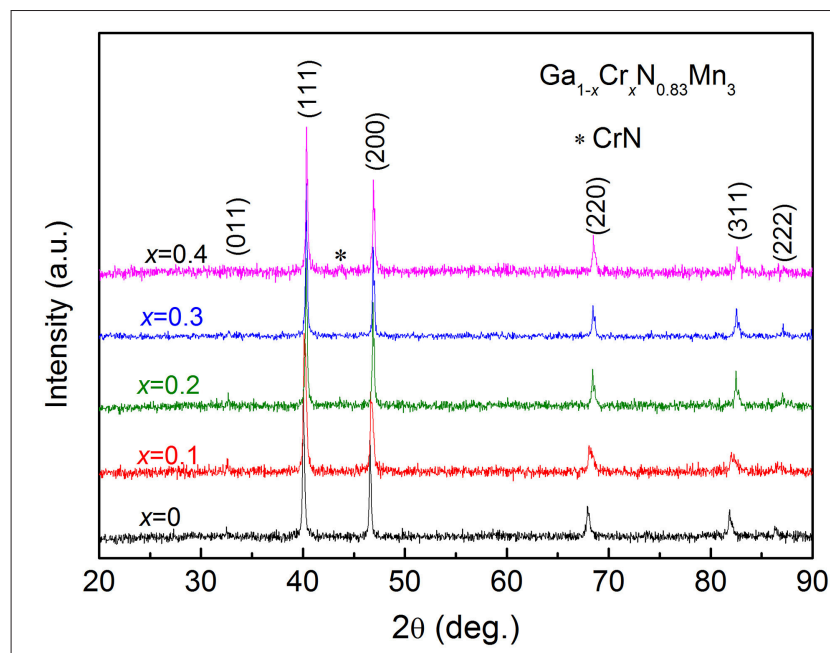

FIGURE 1 | $X$-ray diffractions at room temperature for $\mathrm{Ga}_{1-x} \mathrm{Cr}_{x} \mathrm{~N}_{0.83} \mathrm{Mn}_{3}$ $(x=0,0.1,0.2,0.3,0.4)$. The asterisk marks the diffractions from $\mathrm{CrN}$.

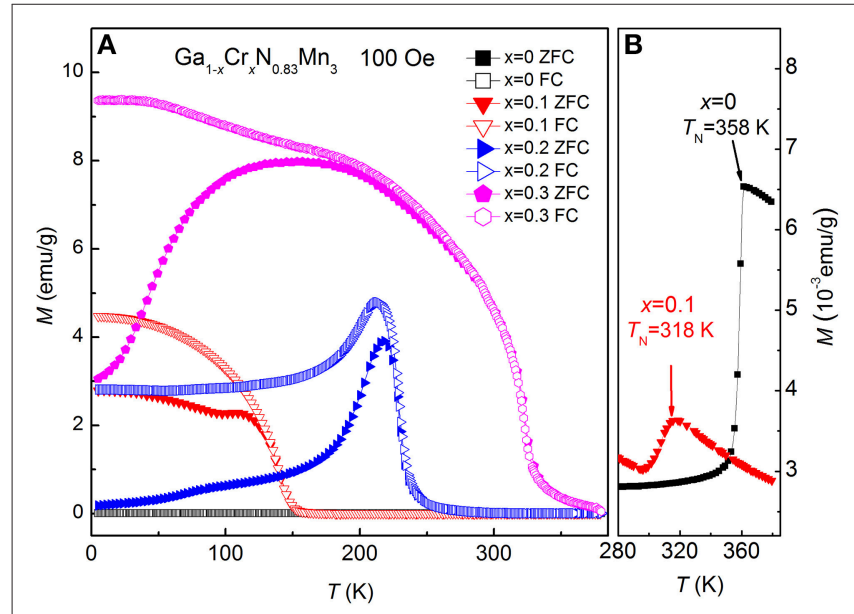

FIGURE 2 | (A) The magnetization $M(T)$ measured at both zero-field-cooling (ZFC) and field-cooling (FC) modes for $\mathrm{Ga}_{1-x} \mathrm{Cr}_{x} \mathrm{~N}_{0.83} \mathrm{Mn}_{3}(x=0,0.1,0.2$, 0.3). (B) shows an enlargement of the high-temperature ZFC data for $x=0$ and 0.1 , where the antiferromagnetic to paramagnetic transition at $T_{N}$ is marked in each curve.

curve deviates from the ZFC one at low temperatures. Figure 3 shows the isothermal hysteresis loop $M(H)$ s at $5 \mathrm{~K}$ for $x=0-0.3$ samples. The magnetization at $45 \mathrm{kOe}\left(M_{45 \mathrm{kOe}}\right)$ increases quickly and linearly with increasing $x$ (inset of Figure 3). At the same time the slopes of $M(H)$ curves at high magnetic fields become smaller as $x$ increases, indicating the FM component is enhanced at the expense of AFM component. For $x=0.3$, a FM ground state is established.

Figure 4 shows the linear thermal expansion $\Delta L / L(380 \mathrm{~K})$ for $\mathrm{Ga}_{1-x} \mathrm{Cr}_{x} \mathrm{~N}_{0.83} \mathrm{Mn}_{3}(0.1 \leq x \leq 0.4)$. Because of the large volume change at $T_{\mathrm{N}}$ which is above room temperature, the as-prepared $\mathrm{GaN}_{0.83} \mathrm{Mn}_{3}$ sample was brittle and thus not subjected to the strain gauge measurement. As shown in Figure 4, at $x=0.1$, the

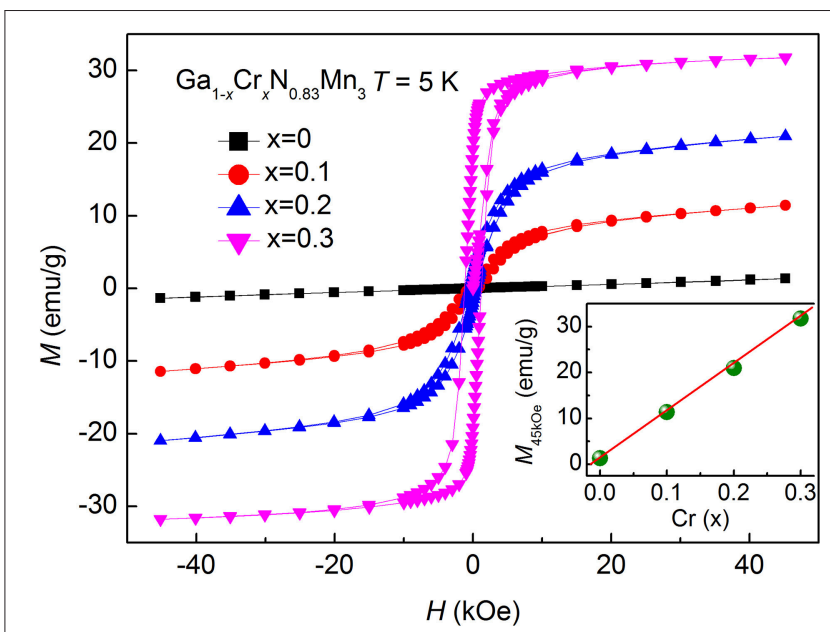

FIGURE 3 | The isothermal magnetization $M(H)$ loops at $5 \mathrm{~K}$ for

$\mathrm{Ga}_{1-x} \mathrm{Cr}_{x} \mathrm{~N}_{0.83} \mathrm{Mn}_{3}(x=0,0.1,0.2,0.3)$ measured between -45 and 45 $\mathrm{kOe}$. Inset shows the magnetization at $45 \mathrm{kOe}, M_{45 \mathrm{kOe}}$, as a function of $\mathrm{Cr}$ content $(x)$.

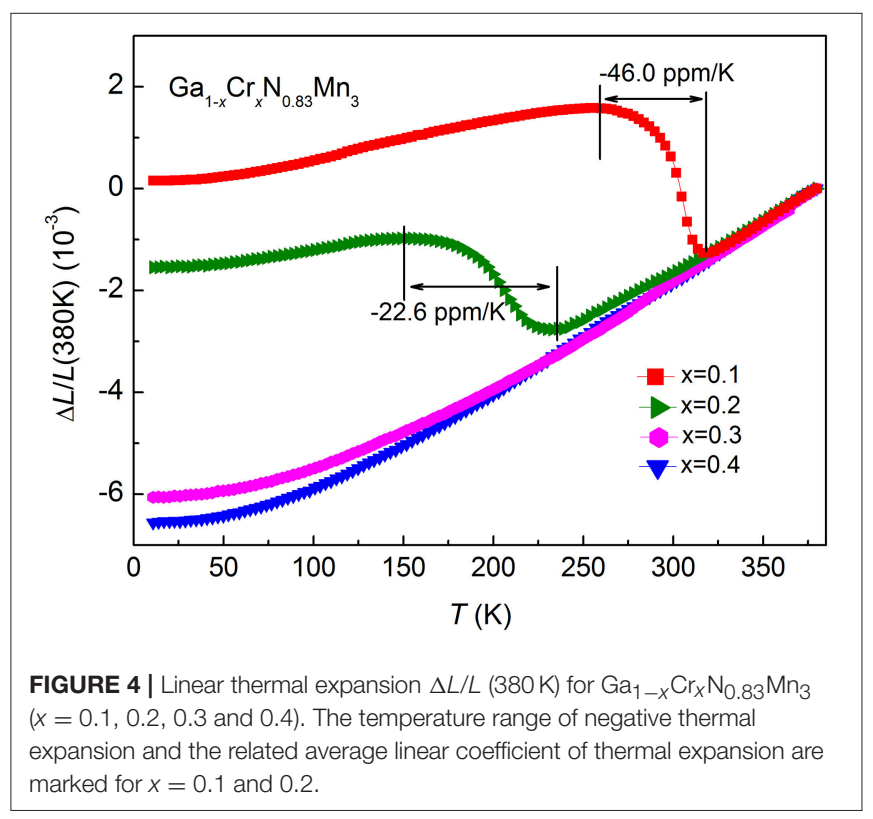

lattice undergoes a continuous shrinkage upon heating between 256 and $318 \mathrm{~K}(\Delta T=62 \mathrm{~K})$ with an average $\alpha_{L}=-46 \mathrm{ppm} / \mathrm{K}$. The onset temperature of NTE region is consistent with the broad AFM transition shown in Figure 2B. For $x=0.2$, The NTE temperature window shifts to $151-232 \mathrm{~K}(\Delta T=81 \mathrm{~K})$, and the corresponding average $\alpha_{L}$ is about $\sim-22.6 \mathrm{ppm} / \mathrm{K}$. The lattice contraction coincides well with the drop of magnetization displayed in both ZFC and FC $M(T)$ s as shown in Figure 2A. When $x$ is further increased $(\geq 0.3)$, no NTE was observed down to $5 \mathrm{~K}$.

Among the many ordered spin configurations, the $\Gamma^{5 \mathrm{~g}}$-type AFM one is special because it adopts a larger lattice volume relative to the $\mathrm{PM}$ or FM state, which is considered as the 
prerequisite for the showing up of NTE (Takenaka et al., 2014). The $\Gamma^{5 \mathrm{~g}}$-type $\mathrm{AFM}$ order is the ground state below $T_{\mathrm{N}}$ for the $x<0.2$ compounds (Kasugai et al., 2012). Most likely, this particular AFM order is involved below $210 \mathrm{~K}$ in $x=0.2$ sample, as manifested by the drop of the magnetization shown in Figure 2A. Upon doping with $\mathrm{Cr}$, the FM order emergences and becomes increasingly strong with $x$, as revealed by enhanced $T_{\mathrm{C}}$ and the low-temperature magnetization. The strengthened FM phase would impede the growth and propagation of AFM order upon cooling probably via the magnetically coupled AFM/FM interfaces (Guo et al., 2015). When $x$ is increased to 0.3, the FM phase is overwhelmingly strong so that the MVE associated with the AFM ordering is no longer able to influence the overall thermal expansion. As a result, the $x=0.3$ compound displays a normal PTE.

The parent compound of $\mathrm{Ga}_{1-x} \mathrm{Mn}_{x} \mathrm{~N}_{0.8} \mathrm{Mn}_{3}$ is very close to that of the current solid solutions in terms of the chemical composition and the value of $T_{\mathrm{N}}$. However, $\mathrm{Cr}$ doping is more effective in disturbing the AFM order and consequently in expanding the temperature range of lattice contraction relative to $\mathrm{Mn}$ doping. For example, with $20 \% \mathrm{Cr}$ doping the $\Delta T$ of NTE is about $80 \mathrm{~K}$, which is even larger than that of $30 \%$ Mn doped sample ( $\Delta T=73 \mathrm{~K}$; Guo et al., 2015). As shown in the inset of Figure 3, $M_{45 \mathrm{kOe}}$ at $5 \mathrm{~K}$ increases linearly with $\mathrm{Cr}$ doping level and reaches $31.8 \mathrm{emu} / \mathrm{g}$ for $x=0.3$. But, for $\mathrm{Ga}_{1-x} \mathrm{Mn}_{x} \mathrm{~N}_{0.8} \mathrm{Mn}_{3}$ the value of $\mathrm{M}_{45 \mathrm{kOe}}$ at $5 \mathrm{~K}$ shows a tendency toward saturation with increasing $x$, and the related value for $x=0.3$ is only $22.3 \mathrm{emu} / \mathrm{g}$ (Guo et al., 2015). Such a difference indicates the more rapid strengthening of FM order in Cr-doped compounds than in $\mathrm{Mn}$-doped ones. So the AFM phase in $\mathrm{Cr}$ doped sample experienced a stronger impendence from the more rapidly developing FM order, leading to a wider NTE window relative to Mn-doped compounds at the same doping level.

Figure 5 shows the specific heat $C_{p}(T)$ for $\mathrm{GaN}_{0.83} \mathrm{Mn}_{3}$ and $\mathrm{Ga}_{0.8} \mathrm{Cr}_{0.2} \mathrm{~N}_{0.83} \mathrm{Mn}_{3}$ between 6 and $245 \mathrm{~K}$. A broad peak was

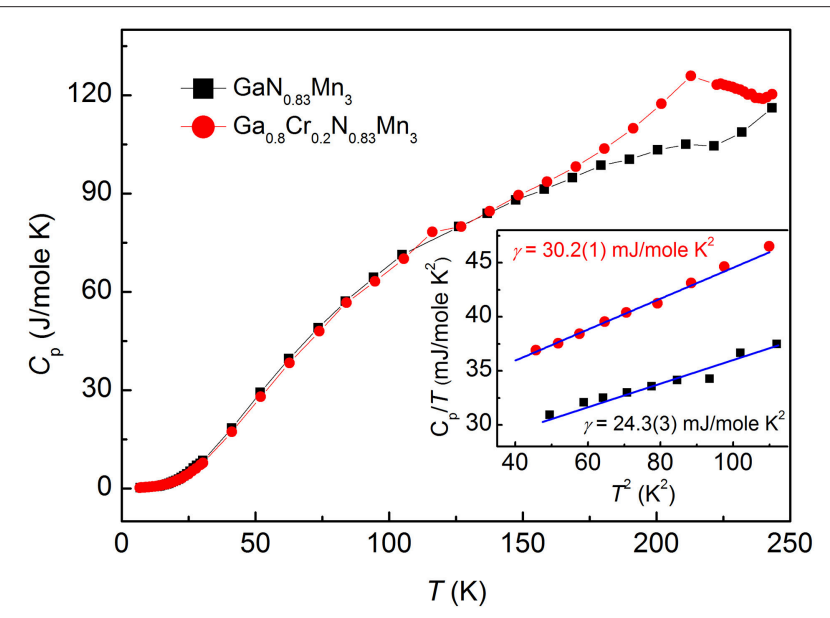

FIGURE 5 | Specific heat $C_{p}(T)$ for $\mathrm{Ga}_{1-x} \mathrm{Cr}_{x} \mathrm{~N}_{0.83} \mathrm{Mn}_{3}$ with $x=0$ and 0.2. Inset shows a linear fit to the $C_{p}(T) / T$ vs. $T^{2}$ curves at low temperatures. The fitted electronic coefficients of specific heat ( $\gamma$, the Sommerfeld constant) are shown for both compounds. observed at $220 \mathrm{~K}$ for $x=0.2$ compound, which is resulted from the structural transition (i.e., the NTE) observed in Figure 4. As shown in the inset of Figure 5, the low-temperature specific heat data for each compound plotted as $C_{p}(T) / T$ vs. $T^{2}$ can be wellfitted linearly by using the expression, $C_{p}(T) / T=\gamma+\beta T^{2}$, where $\gamma$ (i.e., the Sommerfeld constant) represents for the electronic contribution, the second term is the lattice contribution based on the Debye approximation (Wang et al., 2010). The fitted values of $\gamma$ are equal to 24.3(3) and 30.2(1) $\mathrm{mJ} /\left(\mathrm{mol} \mathrm{K}^{2}\right)$ for $\mathrm{GaN}_{0.83} \mathrm{Mn}_{3}$ and $\mathrm{Ga}_{0.8} \mathrm{Cr}_{0.2} \mathrm{~N}_{0.83} \mathrm{Mn}_{3}$, respectively. The value of $\gamma$ corresponds to the density of the electronic DOS at $\mathrm{E}_{\mathrm{F}}$ in the ground state. The enhanced $\gamma$ in the Cr-doped compound indicates an enhancement of DOS at $\mathrm{E}_{\mathrm{F}}$. According the Stoner criterion, FM interactions are enhanced in Cr-doped compound compared with the parent compound (Wang et al., 2010). This may explain why $\mathrm{Cr}$ doping suppresses the AFM ground state and finally changes the background to FM in $x=0.3$ compound. According to the result reported by Garica, the $\gamma$ is remarkably suppressed when the PM state transforms to AFM phase in $\mathrm{GaNMn}_{3}$ (Garcia et al., 1980), which suggests again that the increased DOS at $\mathrm{E}_{\mathrm{F}}$ is not beneficial to the stabilization of AFM ground state of $\mathrm{GaN}_{0.83} \mathrm{Mn}_{3}$.

Although there are no theoretical reports on the electronic structure of Cr-doped $\mathrm{GaNMn}_{3}$, studies on $\mathrm{GaNMn}_{3}$ and $\mathrm{Mn}_{4} \mathrm{~N}$ may give some hints of understanding the magnetism of current compounds. In $\mathrm{GaNMn}_{3}$, all Mn atoms locate at the face centers of the cubic lattice. The hybridized Mn 3d states with N 2p orbitals contribute mainly to the DOS at $\mathrm{E}_{\mathrm{F}}$ (Miao et al., 2005). But Ga contributes little to the overall DOS at $\mathrm{E}_{\mathrm{F}}$ (Miao et al., 2005). However, as to $\mathrm{Mn}_{4} \mathrm{~N}$, the corner $\mathrm{Mn}$ atoms (MnI) contribute a lot to the DOS at $\mathrm{E}_{\mathrm{F}}$, while contribution from the face-center $\mathrm{Mn}$ atoms (MnII) is very similar to that in $\mathrm{GaNMn}_{3}$ (Miao et al., 2005). In $\mathrm{Mn}_{4} \mathrm{~N}$ the magnetic moments at MnI $(3.5 \mu \mathrm{B})$ are antiparallel to those at MnII $(0.9 \mu \mathrm{B})$, leading to a ferrimagnetic ground state below $756 \mathrm{~K}$ (Takei et al., 1962). So $\mathrm{MnI}$ atoms play a dominant role in determining the magnetic properties of $\mathrm{Mn}_{4} \mathrm{~N}$. Analogously, when Cr elements occupy the corner sites (i.e., Ga sites) of the $\mathrm{GaN}_{0.83} \mathrm{Mn}_{3}$, their $3 \mathrm{~d}$ orbitals will contribute to the DOS at $\mathrm{E}_{\mathrm{F}}$. So the substitution of $\mathrm{Cr}$ for $\mathrm{Ga}$ introduces extra d electrons to the system, and thus increases the DOS at $\mathrm{E}_{\mathrm{F}}$, leading to the enhanced FM interactions. A thorough theoretical study on the electronic band structures is needed in order to shed lights on the differences of magnetism and thermal expansion between $\mathrm{Cr}$ and $\mathrm{Mn}$ doped compounds.

\section{CONCLUSIONS}

In summary, we report large NTE at low temperatures in antiperovskite compounds $\mathrm{Ga}_{1-x} \mathrm{Cr}_{x} \mathrm{~N}_{0.83} \mathrm{Mn}_{3}(0 \leq x \leq 0.3)$. With increasing $x$, the NTE window was expanded and moved to lower temperatures quickly. For $x=0.1$ and $x=0.2$, the NTE occurs at $256-318 \mathrm{~K}(\Delta T=62 \mathrm{~K})$ and $151-232 \mathrm{~K}(\Delta T=81 \mathrm{~K})$ with an average $\alpha_{L}$ of -46 and $-22.6 \mathrm{ppm} / \mathrm{K}$, respectively. Finally, for $x \geq 0.3$, NTE was not observed down to $5 \mathrm{~K}$. As revealed by the specific heat measurement, $\mathrm{Cr}$ doping increases the DOS at $\mathrm{E}_{\mathrm{F}}$, which favors the emergence of FM order against 
the AFM background. The competing FM order was suggested to suppress the original AFM order and hinder its propagation upon cooling, leading to the NTE with wide $\Delta T$.

\section{AUTHOR CONTRIBUTIONS}

XG and PT designed the synthetic work; XG carried out the synthesis and characterization of all the compounds; JL, CY, $\mathrm{KZ}$, and SL participated in characterization of structure and magnetism; WS and XG carried out the heat capacity; XG and PT analyzed the data and wrote the manuscript; PT revised the paper; YS did discussion for this work. All authors listed,

\section{REFERENCES}

Azuma, M., Chen, W. T., Seki, H., Czapski, M., Olga, S., Oka, K., et al. (2011). Colossal negative thermal expansion in $\mathrm{BiNiO}_{3}$ induced by intermetallic charge transfer. Nat. Commun. 2:347. doi: 10.1038/ncomms1361

Chen, J., Fan, L. L., Ren, Y., Pan, Z., Deng, J. X., Yu, R. B., et al. (2013). Unusual transformation from strong negative to positive thermal expansion in $\mathrm{PbTiO}_{3}-\mathrm{BiFeO}_{3}$ perovskite. Phys. Rev. Lett. 110:115901. doi: 10.1103/PhysRevLett.110.115901

Chen, J., Hu, L., Deng, J., and Xing, X. (2015). Negative thermal expansion in functional materials: controllable thermal expansion by chemical modifications. Chem. Soc. Rev. 44, 3522-3567. doi: 10.1039/C4CS00461B

Dai, Y. J., Song, X. Y., Huang, R. J., Li, L. F., and Sun, Z. H. (2014). Effect of Si doping on structure, thermal expansion and magnetism of antiperovskite manganese nitrides $\mathrm{Mn}_{3} \mathrm{Cu}_{1-x} \mathrm{Si}_{x} \mathrm{~N}$. Mater. Lett. 139, 409-413. doi: 10.1016/j.matlet.2014.10.136

Deng, S. H., Sun, Y., Wang, L., Shi, Z. X., Wu, H., Huang, Q. Z., et al. (2015a). Frustrated triangular magnetic structures of $\mathrm{Mn}_{3} \mathrm{ZnN}$ : applications in thermal expansion. J. Phys. Chem. C 119, 24983-24990. doi: 10.1021/acs.jpcc.5b07225

Deng, S. H., Sun, Y., Wu, H., Huang, Q. Z., Yan, J., Shi, K. W., et al. (2015b). Invarlike behavior of antiperovskite $\mathrm{Mn}_{3+x} \mathrm{Ni}_{1-x} \mathrm{~N}$ compounds. Chem. Mater. 27, 2495-2501. doi: 10.1021/cm504702m

Fruchart, D., and Bertaut, E. F. (1978). Magnetic studies of the metallic perovskite-type compounds of manganese. J. Phys. Soc. Jpn. 44, 781-791. doi: $10.1143 /$ JPSJ.44.781

Garcia, J., Navarro, R., Bartolome, J., Burriel, R., Gonzalez, D., and Fruchart, D. (1980). Specific heat of the cubic metallic perovskites $\mathrm{Mn}_{3} \mathrm{ZnN}$ and $\mathrm{Mn}_{3} \mathrm{GaN}$. J. Magn. Magn. Mater. 15-8, 1155-1156. doi: 10.1016/0304-8853(80)90231-0

Ge, X., Mao, Y., Liu, X., Cheng, Y., Yuan, B., Chao, M., et al. (2016). Negative thermal expansion and broad band photoluminescence in a novel material of $\mathrm{ZrScMo}_{2} \mathrm{VO}_{12}$. Sci. Rep. 6:24832. doi: 10.1038/srep24832

Goodwin, A. L., Calleja, M., Conterio, M. J., Dove, M. T., Evans, J. S. O., Keen, D. A., et al. (2008). Colossal positive and negative thermal expansion in the framework material $\mathrm{Ag}_{3}\left[\mathrm{Co}(\mathrm{CN})_{6}\right]$. Science 319, 794-797. doi: $10.1126 /$ science. 1151442

Greve, B. K., Martin, K. L., Lee, P. L., Chupas, P. J., Chapman, K. W., and Wilkinson, A. P. (2010). Pronounced negative thermal expansion from a simple structure: cubic $\mathrm{ScF}_{3}$. J. Am. Chem. Soc. 132, 15496-15498. doi: $10.1021 /$ ja106711v

Guo, X. G., Lin, J. C., Tong, P., Wang, M., Wu, Y., Yang, C., et al. (2015). Magnetically driven negative thermal expansion in antiperovskite $\mathrm{Ga}_{1-x} \mathrm{Mn}_{x} \mathrm{~N}_{0.8} \mathrm{Mn}_{3}(0.1 \leq x \leq 0.3)$. Appl. Phys. Lett. 107:202406. doi: $10.1063 / 1.4936239$

Guo, X. G., Tong, P., Lin, J. C., Yang, C., Zhang, K., Wang, M., et al. (2017). Large negative thermal expansion in $\left(\mathrm{Ga}_{0.7} \mathrm{Cu}_{0.3}\right)_{1-x} \mathrm{Mn}_{x} \mathrm{NMn}_{3} \quad(x \leq 0.4)$, compensating for the thermal expansion of cryogenic materials. Scr. Mater. 128, 74-77. doi: 10.1016/j.scriptamat.2016.10.002

Hu, L., Chen, J., Xu, J., Wang, N., Han, F., Ren, Y., et al. (2016). Atomic linkage flexibility tuned isotropic negative, zero, and positive thermal expansion in $\mathrm{MZrF}_{6}(\mathrm{M}=\mathrm{Ca}, \mathrm{Mn}, \mathrm{Fe}, \mathrm{Co}, \mathrm{Ni}$, and Zn). J. Am. Chem. Soc. 138, 14530-14533. doi: $10.1021 /$ jacs.6b08746 have made substantial, direct, and intellectual contribution to the work, and approved it for publication.

\section{ACKNOWLEDGMENTS}

This work was supported by the National Natural Science Foundation of China under contract Nos. U1632158 and 51322105, the Key Research Program of Frontier Sciences, CAS (QYZDB-SSW-SLH015), the Doctoral Initial Funding of Hebei University of Science and Technology (Grant No. 1181297), and the Natural Science Foundation of Hebei Province (Grant No. A2018208071).

Huang, R. J., Li, L. F., Cai, F. S., Xu, X. D., and Qian, L. H. (2008). Low-temperature negative thermal expansion of the antiperovskite manganese nitride $\mathrm{Mn}_{3} \mathrm{CuN}$ codoped with Ge and Si. Appl. Phys. Lett. 93:081902. doi: 10.1063/1.2970998

Huang, R. J., Liu, Y. Y., Fan, W., Tan, J., Xiao, F. R., Qian, L. H., et al. (2013). Giant negative thermal expansion in $\mathrm{NaZn}_{13}$-Type $\mathrm{La}(\mathrm{Fe}, \mathrm{Si}, \mathrm{Co})_{13}$ Compounds. J. Am. Chem. Soc. 135, 11469-11472. doi: 10.1021/ja405161z

Iikubo, S., Kodama, K., Takenaka, K., Takagi, H., and Shamoto, S. (2008a). Magnetovolume effect in $\mathrm{Mn}_{3} \mathrm{Cu}_{1-x} \mathrm{Ge}_{x} \mathrm{~N}$ related to the magnetic structure: neutron powder diffraction measurements. Phys. Rev. B 77:020409. doi: 10.1103/PhysRevB.77.020409

Iikubo, S., Kodama, K., Takenaka, K., Takagi, H., Takigawa, M., and Shamoto, S. (2008b). Local lattice distortion in the giant negative thermal expansion material $\mathrm{Mn}_{3} \mathrm{Cu}_{1-x} \mathrm{Ge}_{x} \mathrm{~N}$. Phys. Rev. Lett. 101:205901. doi: 10.1103/PhysRevLett.101.205901

Jiang, X., Molokeev, M. S., Gong, P., Yang, Y., Wang, W., Wang, S., et al. (2016). Near-zero thermal expansion and high ultraviolet transparency in a borate crystal of $\mathrm{Zn}_{4} \mathrm{~B}_{6} \mathrm{O}_{13}$. Adv. Mater. 28, 7936-7940. doi: 10.1002/adma.201601816

Kasugai, D., Ozawa, A., Inagaki, T., and Takenaka, K. (2012). Effects of nitrogen deficiency on the magnetostructural properties of antiperovskite manganese nitrides. J. Appl. Phys. 111:07E314. doi: 10.1063/1.3672243

Li, B., Luo, X. H., Wang, H., Ren, W. J., Yano, S., Wang, C. W., et al. (2016). Colossal negative thermal expansion induced by magnetic phase competition on frustrated lattices in Laves phase compound (Hf,Ta)Fe 2 . Phys. Rev. B 93:224405. Doi:10.1103/PhysRevB.93.224405

Li, S. P., Huang, R. J., Li, W., Wang, W., Zhao, Y. Q., and Li, L. F. (2015). Low-temperature negative thermal expansion behavior of $\mathrm{LaFe}_{11.2} \mathrm{Al}_{1.8-x} \mathrm{Si}_{x}$ compounds. J. Alloys Compd. 646, 119-123. doi: 10.1016/j.jallcom.2015.05.274

Lin, J. C., Tong, P., Tong, W., Lin, S., Wang, B. S., Song, W. H., et al. (2015). Tunable negative thermal expansion related with the gradual evolution of antiferromagnetic ordering in antiperovskite manganese nitrides $\mathrm{Ag}_{1-x} \mathrm{NMn}_{3+x}(0 \leq x \leq 0.6)$. Appl. Phys. Lett. 106:082405. doi: $10.1063 / 1.4913663$

Lin, J. C., Tong, P., Zhang, K., Tong, H. Y., Guo, X. G., Yang, C., et al. (2016). Colossal negative thermal expansion with an extended temperature interval covering room temperature in fine-powdered $\mathrm{Mn}_{0.98} \mathrm{CoGe}$. Appl. Phys. Lett. 109:241903. doi: 10.1063/1.4972234

Long, Y. W., Hayashi, N., Saito, T., Azuma, M., Muranaka, S., and Shimakawa, Y. (2009). Temperature-induced A-B intersite charge transfer in an A-site-ordered $\mathrm{LaCu}_{3} \mathrm{Fe}_{4} \mathrm{O}_{12}$ perovskite. Nature 458, 60-63. doi: 10.1038/nature07816

Mary, T. A., Evans, J. S. O., Vogt, T., and Sleight, A. W. (1996). Negative thermal expansion from 0.3 to 1050 Kelvin in $\mathrm{ZrW}_{2} \mathrm{O}_{8}$. Science 272, 90-92. doi: $10.1126 /$ science. 272.5258 .90

Matsuno, J., Takenaka, K., Takagi, H., Matsumura, D., Nishihata, Y., and Mizuki, J. (2009). Local structure anomaly around $\mathrm{Ge}$ dopants in $\mathrm{Mn}_{3} \mathrm{Cu}_{0.7} \mathrm{Ge}_{0.3} \mathrm{~N}$ with negative thermal expansion. Appl. Phys. Lett. 94:181904. doi: 10.1063/1.31 29169

Miao, M., Herwadkar, A., and Lambrecht, W. (2005). Electronic structure and magnetic properties of $\mathrm{Mn}_{3} \mathrm{GaN}$ precipitates in $\mathrm{Ga}_{1-x} \mathrm{Mn}_{x} \mathrm{~N}$. Phys. Rev. B 72:033204. doi: 10.1103/PhysRevB.72.033204

Panda, M. K., Runcevski, T., Sahoo, S. C., Belik, A. A., Nath, N. K., Dinnebier, R. E., et al. (2014). Colossal positive and negative thermal expansion 
and thermosalient effect in a pentamorphic organometallic martensite. Nat. Commun. 5:4811. doi: 10.1038/ncomms5811

Romao, C. P., Miller, K. J., Whitman, C. A., White, M. A., and Marinkovic, B. A. (2003). "Negative thermal expansion (thermomiotic) materials", in Comprehensive Inorganic Chemistry, II, eds J. Reedijk and K. Poeppelmeier (Amsterdam: Elsevier), 127-151.

Song, X., Sun, Z., Huang, Q., Rettenmayr, M., Liu, X., Seyring, M., et al. (2011). Adjustable zero thermal expansion in antiperovskite manganese nitride. Adv. Mater. 23:4690. doi: 10.1002/adma.2011 02552

Sun, Y., Wang, C., Wen, Y. C., Chu, L. H., Nie, M., and Liu, F. S. (2010a). Negative thermal expansion and correlated magnetic and electrical properties of Si-doped $\mathrm{Mn}_{3} \mathrm{GaN}$ compounds. J. Am. Ceram. Soc. 93, 650-653. doi: 10.1111/j.1551-2916.2009.03482.x

Sun, Y., Wang, C., Wen, Y. C., Chu, L. H., Pan, H., and Nie, M. (2010b). Negative thermal expansion and magnetic transition in anti-perovskite structured $\mathrm{Mn}_{3} \mathrm{Zn}_{1-x} \mathrm{Sn}_{x} \mathrm{~N}$ Compounds. J. Am. Ceram. Soc. 93, 2178-2181. doi: $10.1111 /$ j.1551-2916.2010.03711.x

Sun, Y., Wang, C., Wen, Y. C., Zhu, K. G., and Zhao, J. T. (2007). Lattice contraction and magnetic and electronic transport properties of $\mathrm{Mn}_{3} \mathrm{Zn}_{1-x} \mathrm{Ge}_{x} \mathrm{~N}$. Appl. Phys. Lett. 91:231913. doi: 10.1063/1.28 22813

Takei, W. J., Heikes, R. R., and Shirane, G. (1962). Magnetic structure of $\mathrm{Mn}_{4} \mathrm{~N}$-type compounds. Phys. Rev. 125:1893. doi: 10.1103/PhysRev.12 5.1893

Takenaka, K., Asano, K., Misawa, M., and Takagi, H. (2008). Negative thermal expansion in Ge-free antiperovskite manganese nitrides: tin-doping effect. Appl. Phys. Lett. 92:011927. doi: 10.1063/1.2831715

Takenaka, K., Ichigo, M., Hamada, T., Ozawa, A., Shibayama, T., Inagaki, T., et al. (2014). Magnetovolume effects in manganese nitrides with antiperovskite structure. Sci. Technol. Adv. Mater. 15:015009. doi: 10.1088/1468-6996/15/1/015009

Takenaka, K., and Takagi, K. (2005). Giant negative thermal expansion in Ge-doped anti-perovskite manganese nitrides. Appl. Phys. Lett. 87:261902. doi: 10.1063/1.2147726

Tan, J., Huang, R. J., Li, W., Han, Y. M., and Li, L. F. (2014). Broadened negative thermal expansion operation-temperature window in antiperovskite
$\mathrm{Mn}_{3} \mathrm{Zn}_{0.6} \mathrm{Ge}_{0.4} \mathrm{~N}$ prepared by spark plasma sintering. J. Alloys Compd. 593, 103-105. doi: 10.1016/j.jallcom.2014.01.027

Tong, P., Louca, D., King, G., Llobet, A., Lin, J. C., and Sun, Y. P. (2013a). Magnetic transition broadening and local lattice distortion in the negative thermal expansion antiperovskite $\mathrm{Cu}_{1-x} \mathrm{Sn}_{x} \mathrm{NMn}_{3}$. Appl. Phys. Lett. 102:041908. doi: $10.1063 / 1.4790151$

Tong, P., Wang, B. S., and Sun, Y. P. (2013b). Mn-based antiperovskite functional materials: review of research. Chin. Phys. B 22:067501. doi: 10.1088/1674-1056/22/6/067501

Wang, B. S., Li, C. C., Lin, J. C., Lin, S., Tong, P., Zhu, X. B., et al. (2010). Metastability across the antiferromagnetic-ferromagnetic intermediate phase transition and enhanced giant magnetoresistance in $\mathrm{Zn}$-doped antiperovskite compounds $\mathrm{Ga}_{1-x} \mathrm{Zn}_{x} \mathrm{CMn}_{3}$. Appl. Phys. Lett. 97:142505. doi: $10.1063 / 1.3499216$

Xing, X. R., Chen, J., Deng, J. X., and Liu, G. R. (2003). Solid solution $\mathrm{Pb}_{1-x} \mathrm{Sr}_{x} \mathrm{TiO}_{3}$ and its thermal expansion. J. Alloys Compd. 360, 286-289. doi: 10.1016/S0925-8388(03)00345-1

Yamada, I., Tsuchida, K., Ohgushi, K., Hayashi, N., Kim, J., Tsuji, N., et al (2011). Giant negative thermal expansion in the iron perovskite $\mathrm{SrCu}_{3} \mathrm{Fe}_{4} \mathrm{O}_{12}$. Angew Chem. Int. Ed. Engl. 50, 6579-6582. doi: 10.1002/anie.2011 02228

Zhao, Y. Y., Hu, F. X., Bao, L. F., Wang, J., Wu, H., Huang, Q. Z., et al. (2015). Giant negative thermal expansion in bonded MnCoGe-Based compounds with $\mathrm{Ni}_{2}$ In-Type hexagonal structure. J. Am. Chem. Soc. 137, 1746-1749. doi: $10.1021 /$ ja510693a

Conflict of Interest Statement: The authors declare that the research was conducted in the absence of any commercial or financial relationships that could be construed as a potential conflict of interest.

Copyright (๑) 2018 Guo, Tong, Lin, Yang, Zhang, Lin, Song and Sun. This is an open-access article distributed under the terms of the Creative Commons Attribution License (CC BY). The use, distribution or reproduction in other forums is permitted, provided the original author(s) and the copyright owner are credited and that the original publication in this journal is cited, in accordance with accepted academic practice. No use, distribution or reproduction is permitted which does not comply with these terms. 